\title{
A relação direta entre vitamina $D$ e insuficiência cardíaca: Uma revisão sistemática.
}

Suzane Medeiros Bacelar (1), Irlan Fernandes Bacelar (2) e Salomão Barauna Alcolumbre (3)

\begin{abstract}
ARTIGO DE REVISÃO
Resumo

Introducão: A insuficiência cardíaca (IC) é um problema de saúde pública de primeira ordem, caracterizado por alta mortalidade, hospitalizações frequentes e deterioração da qualidade de vida, com prevalência e incidência que estão aumentando em todo o mundo. A vitamina $D$ é um micronutriente importante para a função óptima de muitos órgãos e tecidos por todo o corpo, incluindo o sistema cardiovascular.
\end{abstract}

Objetivo: O objetivo deste artigo é revisar a relação entre insuficiência cardíaca e a vitamina D.

Metodologia: Foi realizada uma varredura nas bases de indexação Google Scholar e Scopus. Os artigos referentes a insuficiência cardíaca e vitamina $D$ foram selecionados e analisados quanto a sua qualidade e relevância.

Conclusão: Foi sugerido que a deficiência de vitamina $D$ pode ser um fator importante tanto na gênese dos fatores de risco quanto nas doenças cardiovasculares.

Palavras chave: Vitamina D, Insuficiência Cardíaca, Saúde Pública.

Instituição afiliada 1-Implantodontista, Endodontista e Ortodontista no Departamento de Odontologia da Odontocardio. 2- Médico Cardiologista no Departamento de Cardiologia da Odontocardio. 3 -Médico Cardiologista no Departamento de Cardiologia NEUROCOR.

Dados da publicação: Article received on June 09, revised on June 17, accepted for publication on June 25 and published on June 29.

DOI: https://doi.org/10.36557/2674-8169.2020v2n6p34-51

@- Suzane Medeiros Bacelar. dentista-suzanemedeiros@hotmail.com 


\section{The direct relationship between vitamin $D$ and heart failure: $A$ systematic review.}

Introduction: Heart failure (HF) is a public health problem of the highest order, characterized by high mortality, frequent hospitalizations and deteriorating quality of life, with prevalence and incidence that are increasing worldwide. Vitamin D is an important micronutrient for the optimal function of many organs and tissues throughout the body, including the cardiovascular system.

Objective: The purpose of this article is to review the relationship between heart failure and vitamin D.

Methodology: A scan was performed on the Google Scholar and Scopus indexing bases. Articles on heart failure and vitamin D were selected and analyzed for quality and relevance.

Conclusion: It has been suggested that vitamin D deficiency may be an important factor in both the genesis of risk factors and cardiovascular diseases.

Keywords: Vitamin D, Heart Failure, Public Health..

Instituição afiliada 1-Implantodontista, Endodontista e Ortodontista no Departamento de Odontologia da Odontocardio. 2- Médico Cardiologista no Departamento de Cardiologia da Odontocardio. 3 -Médico Cardiologista no Departamento de Cardiologia NEUROCOR.

Dados da publicação: Article received on June 09, revised on June 17, accepted for publication on June 25 and published on June 29.

DOI: https://doi.org/10.36557/2674-8169.2020v2n6p34-51

Suzane Medeiros Bacelar. dentista-suzanemedeiros@hotmail.com 


\section{INTRODUÇÃO}

A insuficiência cardíaca (IC) é um problema de saúde pública de primeira ordem, caracterizado por alta mortalidade, hospitalizações frequentes e deterioração da qualidade de vida, com prevalência e incidência que estão aumentando em todo o mundo [1,2] . Embora o prognóstico tenha melhorado nas últimas décadas, graças à melhora diagnóstica e terapêutica das doenças cardiovasculares, a morbimortalidade desses pacientes permanece alta [3] . Tudo isso significa que novas metas e opções de tratamento ainda são necessárias.

Tradicionalmente, a vitamina $\mathrm{D}$ havia sido associada apenas à saúde óssea, e foi aceito que a deficiência de vitamina $D$ causava osteomalácia e osteoporose em adultos e raquitismo em crianças $[4,5]$. No entanto, os dados obtidos nos anos recentes indicam que a vitamina D é um micronutriente importante para a função óptima de muitos órgãos e tecidos por todo o corpo, incluindo o sistema cardiovascular $[6,7]$. Foi sugerido que a deficiência de vitamina $D$ pode ser um fator importante tanto na gênese dos fatores de risco quanto nas doenças cardiovasculares 7como marcador prognóstico na IC. Dados fisiopatológicos indicam que a deficiência de vitamina $D$ pode ser muito prejudicial para pacientes com IC e que a abordagem terapêutica com suplementação de vitamina $D$ pode ser potencialmente benéfica, embora tudo isso não seja isento de controvérsias [8] .

Neste artigo, revisamos as evidências de que, até o momento, apoiam a ligação entre a vitamina $\mathrm{D}$ e a $\mathrm{Cl}$, analisamos os mecanismos potenciais pelos quais a vitamina $\mathrm{D}$ poderia exercer seus efeitos cardioprotetores e os efeitos deletérios potenciais de seu déficit, e decompomos o principais estudos sobre suplementação de vitamina D em pacientes com IC.

\section{PATOSFISIOLOGIA DA VITAMINA DA INSUFICIÊNCIA CARDÍACA}

Não existe uma única via estabelecida ou hipótese única que explique a relação entre a vitamina $\mathrm{D}$ e a $\mathrm{Cl}$. O receptor da vitamina $\mathrm{D}$ (RVD) é um receptor hormonal nuclear que medeia a ação do calcitriol por vias genômicas e não-genômicas [9] . Os cardiomiócitos têm RVD, e sabe-se que o calcitriol através do RVD também modula genes importantes relacionados à saúde cardiovascular, podendo ser influenciados pela vitamina D [10] .

Os RVD funcionais são expressos no núcleo celular ou adjacentes aos túbulos T dos cardiomiócitos e também dos fibroblastos cardíacos. A hipertrofia cardíaca tem sido associada ao aumento da expressão desses receptores nessas células. A vitamina $D$ também tem sido atribuída uma propriedade antiproliferativa mediada pela supressão de proto-oncogenes como o c-mic, bem como o peptídeo natriurético, atuando diretamente no crescimento e 
diferenciação dos cardiomiócitos. Camundongos sem RVD (nocaute do gene VDR) apresentam aumento da deposição de colágeno em suas estruturas cardíacas [10] .

Da mesma forma, existem mecanismos moleculares mais complexos que podem explicar a relação entre vitamina $\mathrm{D}$ e $\mathrm{Cl}$. A vitamina $\mathrm{D}$ atua nos canais de cálcio dos cardiomiócitos, induzindo uma rápida entrada de cálcio intracelular [11]. Essa concentração intracelular de cálcio controla respostas de longo prazo ao crescimento, proliferação e morte celular. Além disso, ao ativar a proteína $\mathrm{C}$-cinase, promove o relaxamento dos cardiomiócitos e, portanto, participa da função diastólica cardíaca [12] e da sístole cardíaca, ativando a adenilina ciclase cíclica ou o monofosfato de adenosina. A disfunção de qualquer uma dessas vias pode produzir disfunção ventricular sistólica e / ou diastólica e, consequentemente, $\mathrm{Cl}$.

Vários sistemas neuroendócrinos e citocinas inflamatórias desempenham um papel fundamental na fisiopatologia da vitamina $\mathrm{D}$ e da $\mathrm{Cl}$. Eles são ativados para manter a homeostase circulatória, mas, a longo prazo, contribuem para aumentar a resistência sistêmica e a remodelação ventricular, desenvolvendo e agravando a IC. Embora o sistema renina-angiotensina-aldosterona (SARS) e o sistema nervoso simpático (SNS) tenham sido até agora os mais importantes na IC, recentemente, tanto o diagnóstico como o terapêutico, sistemas contra-reguladores, como peptídeos natriuréticos, também estão sendo essenciais na abordagem diagnóstico-terapêutica dessa síndrome $[13,14]$.

A vitamina $D$ demonstrou ter um relacionamento íntimo com a SARS. Estudos têm mostrado uma correlação inversa entre os níveis de vitamina $D$ e a actividade da RAAS 15 - 17 . As principais ações da SARS incluem a regulação do tônus vascular, volume sanguíneo, remodelação ventricular e vascular e ativação do SNS; O papel principal da SARS na fisiopatologia da IC e pressão alta está bem definido.

A cascata de ação e fisiopatologia da SARS é a seguinte: A renina é uma proteína que atua no angiotensinogênio, produzindo angiotensina I, que é transformada em angiotensina II pela ação da enzima conversora de angiotensina nos níveis pulmonar e vascular. A angiotensina II é um hormônio vasoconstritor potente das arteríolas renais aferentes e eferentes, além de promover a ativação do sistema nervoso simpático (também essencial na fisiopatologia da IC). A superativação do SNS e do SRAA contribui para a remodelação cardíaca progressiva que pode levar à IC. Esse hormônio, por sua vez, também favorece a liberação de aldosterona do córtex adrenal, importante no equilíbrio de eletrólitos e volumes, retendo sódio e água e liberando potássio e magnésio no nível renal [18].

Embora um dos principais fatores que estimulam a liberação de renina e, portanto, a SARS seja a diminuição da perfusão renal, estudos experimentais tenham demonstrado que, após modificar a função do RVD em animais experimentais com camundongos knock-out para RVD, um aumento na a concentração da expressão de renina com aumento do mRNA e de sua proteína no rim e da angiotensina II plasmática, em comparação com camundongos do tipo wil 15 . Consequentemente, esses camundongos eliminados desenvolveram mais pressão alta, 
hipertrofia ventricular esquerda e maior retenção de líquidos. Injeção de 1,25 (OH) 2D alcançou supressão acentuada da renina, que também foi alcançada com o uso do inibidor da enzima de conversão da angiotensina, captopril ou antagonista do receptor da angiotensina II, losartan; Isso mostrou o principal papel fisiopatológico da SARS [15, 19] , mostrando que a causa provável disso é o superestimulação da SARS 20 . O papel da angiotensina II no aumento da fibrose e hipertrofia cardíaca, aumento do tônus vascular e, portanto, pressão arterial, bem como aumento do tônus simpático e uma relação direta com os sintomas e progressão da insuficiência cardíaca em humanos também é claramente estabelecido.

A deficiência de vitamina $D$ tem sido associada ao aumento da produção e liberação de citocinas inflamatórias, que afetam direta e indiretamente o coração e outros órgãos. As citocinas inflamatórias induzem apoptose dos cardiomiócitos, hipertrofia, fibrose, remodelação cardíaca e anormalidades iônicas negativas, como retenção de sódio e, portanto, retenção de líquidos [21]. Também aumenta a atividade catabólica e induz caquexia, o que contribui para a progressão da síndrome da IC [22]. Estudos in vitro sugeriram que a vitamina $D$ inibe citocinas inflamatórias como TNF- $\alpha$ e IL-6, enquanto estimula citocinas antiinflamatórias como IL-10 [23].

Os RVDs também estão presentes na glândula paratireóide e o calcitriol suprime a produção do hormônio da paratireóide (PTH) e evita a proliferação das glândulas paratireóides [24] . Quando há deficiência de vitamina D, ocorre hiperparatireoidismo secundário, o que também leva a efeitos deletérios cardiovasculares e tróficos nos cardiomiócitos. Esse aumento nos níveis de PTH também leva a um aumento da pressão arterial devido ao aumento da rigidez arterial e, portanto, contribui, mais uma vez, para o remodelamento cardíaco na IC secundário à hipertrofia, apoptose e fibrose do ventrículo $[10,25]$.

Outro mecanismo fisiopatológico é a influência da vitamina $D$ na regeneração da matriz extracelular do miocárdio, outra maneira pela qual pode ser prejudicial à estrutura e função cardíaca. Estudos experimentais com camundongos knockout para RVD mostraram que a ausência de vitamina $D$ está associada a um aumento na expressão e atividade das metaloproteinases da matriz miocárdica (MMP), o que resulta em remodelação miocárdica, aumento da deposição de colágeno e aumento da fibrose [26, 27]. A vitamina D modula a regeneração da matriz extracelular do miocárdio, atuando na expressão de metaloproteinases da matriz (MMP) que hidrolisam proteínas da matriz extracelular (MEC) e inibidores teciduais de metaloproteinases (TIMP). Em camundongos knock-out com RVD, a expressão desequilibrada de MMP / TIMP foi caracterizada pela regulação positiva das metaloproteinases de MMP-2 e MMP-9 de TIMP-1 e TIMP-3. O desequilíbrio entre MMP e TIMP promoveu a destruição do tecido miocárdico e a remodelação ventricular; tudo isso está intimamente relacionado aos complexos processos de iniciação e progressão da IC diastólica e sistólica [28]. Deve-se notar também que certas citocinas inflamatórias, como o TNF-alfa, também foram consideradas um importante regulador da atividade da MMP e podem contribuir para essa via fisiopatológica [29] . 
A doença arterial coronariana é um fator importante no desenvolvimento da IC, e a deficiência de vitamina $D$ tem sido associada ao aumento da arteriosclerose e à calcificação das artérias coronárias [1 , 9, 27 , 30]. Esta observação é consistente com a relação inversa entre materializado vitamina $D$ e calcificação das artérias coronárias [6, 30,31$]$. Está documentado que as células endoteliais também expressam RVD e que a vitamina $D$ aumenta a atividade do óxido nítrico in vitro [32] , melhora a produção do fator de crescimento vascular endotelial [33] e reduz a agregação plaquetária endotelial [34] . Finalmente, há evidências de que a deficiência de vitamina $D$ pode ser um importante fator regulador do sistema cardiorrenal. Como destacamos anteriormente, os sistemas cardiovascular e renal estão intimamente relacionados, de forma que alterações no funcionamento de um podem deteriorar progressivamente o outro [34] .

Quando há uma progressão da síndrome cardiorrenal, isso também implica ativação neuro-hormonal, principalmente do sistema renina-angiotensina e do sistema nervoso simpático, e de mecanismos inflamatórios sistêmicos, como descrito anteriormente. Isso influencia mais uma vez a fibrose e a remodelação ventricular, distúrbios hidroeletrolíticos e disfunção cardíaca e renal; desencadeando um circuito vicioso negativo em resposta à deterioração do sistema cardiorrenal, com maior ativação de citocinas inflamatórias e neurohormonais, resultando em aumento da disfunção sistêmica.

Na população com doença renal crônica (DRC), assim como na população com IC, a prevalência de hipovitaminose $D$ é alta e também tem sido associada a um risco aumentado de eventos cardiovasculares [34] . Uma redução na atividade da enzima 1- alfa-hidroxilase e a depleção de proteínas de ligação à vitamina $D$ ao VD secundário à proteinúria são responsáveis pelo fato de os pacientes com DRC serem deficientes em vitamina D. Portanto, a estreita correlação da $\mathrm{Cl}$ e da doença renal crônica destaca a importância da vitamina $\mathrm{D}$ nas patologias e na fisiopatologia da síndrome cardiorrenal.

Tabela 1 Prevalência de deficiência de vitamina D na insuficiência cardíaca (IC) 
A relação direta entre vitamina D e insuficiência cardíaca: Uma revisão sistemática.

Suzane Medeiros Bacelar, Irlan Fernandes Bacelar e Salomão Barauna Alcolumbre

\begin{tabular}{|c|c|c|c|c|c|}
\hline $\begin{array}{l}\text { Primer autor } \\
\text { An̂o } \\
\text { Tipo de estudio }\end{array}$ & $\begin{array}{l}\text { Pacientes } \\
\text { (n) }\end{array}$ & $\begin{array}{c}\text { Criterio } \\
\text { de inclusión }\end{array}$ & $\begin{array}{c}\text { Definición de } \\
\text { hipovitaminosis }\end{array}$ & $\begin{array}{l}\text { Edad media } \\
\text { (años) } \\
\text { /mujeres (\%) }\end{array}$ & Prevalencia \\
\hline $\begin{array}{l}\text { Gostman l et al. } \\
2012 \\
\text { Prospectivo }\end{array}$ & $3.009 / 46.825$ & $\begin{array}{l}\text { Pacientes } 245 \text { años } \\
\text { con IC vs. población } \\
\text { control con } 25 \cdot H C C \\
\text { medida }\end{array}$ & $\begin{array}{c}25 \cdot \mathrm{HCC} \\
<10 \mathrm{ng} / \mathrm{ml}\end{array}$ & $\begin{array}{c}75,9 \pm 10,7 / \\
64,7 / 11,3\end{array}$ & $\begin{array}{l}14,8 \mathrm{ng} / \mathrm{ml} / 16,3 \mathrm{ng} / \mathrm{ml} \\
(28 \% \text { frente } 22 \% \text {, } \\
\text { p<0,00001). En grupo IC, } \\
\text { sólo el } 8,8 \% \text { tenian } \\
25-\mathrm{HCC}>30 \mathrm{ng} / \mathrm{ml}\end{array}$ \\
\hline $\begin{array}{l}\text { Liu L. et al. } \\
2011 \\
\text { Prospectivo }\end{array}$ & 548 & IC NYHA II-IV & $\begin{array}{c}25 \cdot \mathrm{HCC} \\
<20 \mathrm{ng} / \mathrm{ml}\end{array}$ & $74 / 61$ & $75 \%$ cohorte \\
\hline $\begin{array}{l}\text { Kim DH et al. } \\
2008 \\
\text { Transversal }\end{array}$ & 8.351 & $\begin{array}{l}\text { Adultos con niveles } \\
\text { de } 25 \cdot \mathrm{HCC} \text { medidos }\end{array}$ & $\begin{array}{c}25-\mathrm{HCC} \\
<30 \mathrm{ng} / \mathrm{ml}\end{array}$ & & $\begin{array}{l}74 \% \text { de la poblacióngeneral } \\
89 \% \text { de hipovitaminosis D } \\
\text { en pacientes con IC y EAC }\end{array}$ \\
\hline $\begin{array}{l}\text { Zittermann et al. } \\
2003 \\
\text { Transversal }\end{array}$ & $24 / 34 / 34$ & $\begin{array}{l}\text { Pacientes IC NYHA } \\
\text { II-IV }<50 \text { años vx } 250 \\
\text { años vs. control } \\
\operatorname{sin~IC~} 250 \text { años }\end{array}$ & & $38,9 / 64,1 / 68,9$ & $\begin{array}{l}\text { Ambos grupos con IC } \\
\text { presentaron niveles } \\
\text { disminuidos de } 25-\mathrm{HCC} \text { y } \\
\text { calcitriol ( } \mathrm{p}<0,001 \text { ). } \\
\text { Correlación inversa de } \\
\text { niveles de vitamina D con } \\
\text { severidad de IC (valorada } \\
\text { por niveles de Nt-proBNP) }\end{array}$ \\
\hline $\begin{array}{l}\text { Shane E et al. } \\
1997 \\
\text { Transversal }\end{array}$ & 101 & $\begin{array}{l}\text { IC NYHA III-IV; } \\
\text { consideración para } \\
\text { trasplante }\end{array}$ & $\begin{array}{l}25-\mathrm{HCC} \\
59 \mathrm{ng} / \mathrm{ml}\end{array}$ & $\mathrm{NE} / 22$ & $\begin{array}{l}17 \% \text { de hipovitaminosis D } \\
\text { en pacientes con } 25 \cdot \mathrm{HCC} \\
\leq 9 \mathrm{ng} / \mathrm{ml} \mathrm{y} 26 \% \mathrm{con} \\
1,25(\mathrm{OH})_{2} \mathrm{D} \leq 15 \mathrm{pg} / \mathrm{mL} \text {. } \\
\text { Correlación inversa entre } \\
\text { severidad de IC } \mathrm{y} \text { niveles } \\
\text { de vitamina } \mathrm{D}\end{array}$ \\
\hline
\end{tabular}

NYHA: escala da New York Heart Association; DAC: doença coronariana aguda; Nt-proBNP: propeptídeo natriurético cerebral terminal-N; NE: não especificado.

\section{PREVALÊNCIA DE HIPOVITAMINOSE DE INSUFICIÊNCIA CARDÍACA}

Embora não haja consenso sobre os níveis ideais de vitamina $D$, a deficiência desse hormônio é definida pela maioria dos especialistas como um nível de 25- hidroxicolecalciferol (25-HCC) menor que $20 \mathrm{ng} / \mathrm{ml} 4$, [38 - 42] . Para ser mais específico, de acordo com o consenso aceito uniformemente pelas sociedades científicas dedicadas ao metabolismo mineral ósseo, considera-se que os pacientes apresentam níveis ótimos de vitamina $D$ quando os valores séricos de 25-HCC estão acima de $30 \mathrm{ng} / \mathrm{ml}$; considera-se que existe entre 29 e 20 $\mathrm{ng} / \mathrm{ml}$ de insuficiência; e abaixo de $20 \mathrm{ng} / \mathrm{ml}$, a existência de uma deficiência é estabelecida, o que seria grave com níveis de 25-HCC abaixo de $10 \mathrm{ng} / \mathrm{ml}$ [38,41]. Da mesma forma, surgiu a teoria da relação em forma de $U$ entre os níveis de vitamina $D$ e qualquer causa de mortalidade, doenças cardiovasculares, certos tipos de câncer, quedas e fraturas, e que o envenenamento por vitamina D é observado com os níveis séricos 25-HCC $150 \mathrm{ng} / \mathrm{ml}$ [43] . 
Guias de prática clínica orientações recomendo os níveis de vitamina D no plasma não devem rotineiramente medido na população em geral e só deve ser medido em pacientes em populações de risco consideradas para esta deficiência de hormona de [44 - 47] .

Nos últimos anos, foi demonstrado que a deficiência de vitamina $D$ provavelmente foi subestimada e é muito mais prevalente do que o anteriormente reconhecido. Estima- se uma prevalência mundial de um bilhão de indivíduos com níveis insuficientes e insuficientes de vitamina D [42], e foi descrito que $40-80 \%$ da população adulta tem deficiência de vitamina $D$ [48, 49] sendo de particular importância para as mulheres nos países da Oriente Médio. Inúmeros fatores de risco para deficiência de vitamina D foram descritos, como idade, hiperpigmentação da pele, hospitalização em instituições, latitude distante do Equador, obesidade, tabagismo, doença renal, doença hepática ou certos medicamentos como corticosteróides, fenitoína ou fenobarbital [49]. De especial relevância é a epidemia global da obesidade nos países desenvolvidos, que influencia significativamente a deficiência de vitamina $D$, dado o seqüestro de que esse hormônio ocorre no tecido adiposo [48] .

Tabela 2 Níveis de vitamina D e risco de insuficiência cardíaca (IC) 


\begin{tabular}{|c|c|c|c|c|c|}
\hline $\begin{array}{l}\text { Primer autor } \\
\text { Ala } \\
\text { Tipo de estudio }\end{array}$ & $\begin{array}{l}\text { Pacientes } \\
\text { (n) }\end{array}$ & $\begin{array}{c}\text { Criterio } \\
\text { de inclusión }\end{array}$ & $\begin{array}{l}\text { Edad media } \\
\text { (aAios) } \\
\text { Sexo } F(\%)\end{array}$ & Seguimiento & Resultados \\
\hline $\begin{array}{l}\text { Bansal N et al. } \\
2014 \\
\text { Prospectivo }\end{array}$ & 6.469 & $\begin{array}{l}\text { Población general } \\
\text { Libre de ECV } \\
\text { establecida (Multi } \\
\text { Ethnic Study of } \\
\text { Atherosderusis) }\end{array}$ & $\begin{array}{l}62 \\
53\end{array}$ & 8,4 años & 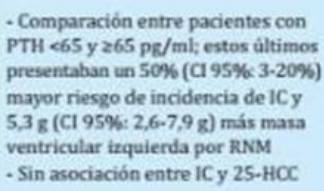 \\
\hline $\begin{array}{l}\text { Wannamethee } \\
\text { SG et al. } \\
2014 \\
\text { Prospectivo }\end{array}$ & 3.713 & $\begin{array}{l}\text { Población general } \\
\text { edad } 60-79 \text { años } \\
\text { con y sin ECV } \\
\text { establecida }\end{array}$ & $\begin{array}{c}68 \\
0\end{array}$ & 13 antos & $\begin{array}{l}\text {-En pacientes con PTH }>55,6 \mathrm{Pg} / \mathrm{ml} \text {, } \\
\text { riesgo de IC de novo del } 1,66 \mathrm{HR} \text { (C } \\
95 \%: 1,30-2,1) \\
\text { - Sin asociación entre los niveles de } \\
25 \text {-HCC, calcio o fosforo con riesgo } \\
\text { de IC (HRa1,07; CI95\%:0,67.1,71) }\end{array}$ \\
\hline $\begin{array}{l}\text { Kestenhaum B } \\
\text { et al } \\
2011 \\
\text { Prospectivo }\end{array}$ & 2.312 & $\begin{array}{c}\text { Sujetos sanos } \\
265 \text { ah̄os }\end{array}$ & $\begin{array}{l}75 \\
\text { NI }\end{array}$ & 14 años & 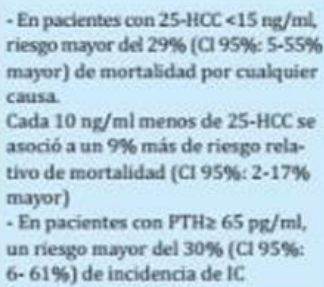 \\
\hline $\begin{array}{l}\text { Pilz Set al } \\
2008 \\
\text { Prospectivo }\end{array}$ & 3.299 & $\begin{array}{l}\text { Pacientes } \\
\text { caucísicos } \\
\text { sometidos a un } \\
\text { cateterismo } \\
\text { cardiaco }\end{array}$ & $\begin{array}{l}63 \\
\mathrm{NI}\end{array}$ & 7,7 affos & 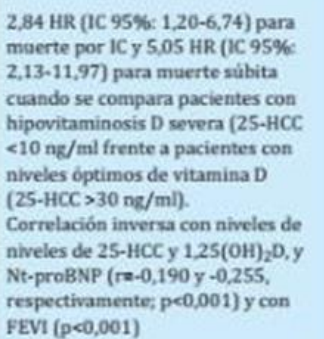 \\
\hline
\end{tabular}

DCV: doença cardiovascular; RMN: ressonância magnética nuclear; NI: não indicado; HR: taxa de risco ; IC: intervalo de confiança; Nt-proBNP: propeptideo natriurético cerebral terminal-N; FEVE: fração de ejeção do ventrículo esquerdo.

Também é verdade que uma deficiência de vitamina $D$ foi observada em uma população jovem e aparentemente saudável , 50 descrita em até aproximadamente $50 \%$ dos adultos jovens; mesmo em estudos realizados em áreas com alta exposição à luz solar, como Ilhas Canárias, Israel, Austrália, Turquia, Índia ou Líbano, onde 30-50\% das crianças e adultos têm níveis de $25-\mathrm{HCC}<20 \mathrm{ng} / \mathrm{ml}$ de [51 - 53] .

Da mesma forma, dados obtidos no National Health and Nutrition Examination Survey (NHANES) encontraram uma prevalência de hipovitaminose D de $74 \%$ na população em geral, aumentando significativamente a prevalência para $89 \%$ quando apenas foram considerados pacientes com IC e doença arterial coronariana. ( odds ratio $[\mathrm{OR}]=3,52$; intervalo de confiança de $95 \%$ [IC]: 1,58-7,84) [54]. Outro estudo, com características semelhantes, no qual foram incluídos 4.105 indivíduos de uma população em geral com pelo menos uma determinação de vitamina $D$, constatou que apenas $36 \%$ dessa coorte apresentava níveis normais de vitamina 
D. Foi observada uma prevalência mais alta de insuficiência cardíaca ( $90 \%$ relativo e $9 \%$ absoluto) em indivíduos com níveis de vitamina $D$ de? $15 \mathrm{ng} / \mathrm{ml}$, e em um seguimento de 1,3 $\pm 1,2$ anos em uma população com mais de 50 anos uma incidência de novos casos de IC foi observada em $2,5 \%$ dessa coorte. Neste estudo, concluiu-se que os níveis plasmáticos de vitamina $D$ têm correlação inversa com o risco de desenvolver IC.

Tabela 3 Impacto prognóstico da deficiência de vitamina D em pacientes com insuficiência cardíaca (IC)

\begin{tabular}{|c|c|c|c|c|c|}
\hline $\begin{array}{l}\text { Primero autor } \\
\text { Año } \\
\text { Tipo de estudío }\end{array}$ & $\begin{array}{l}\text { Pacientes } \\
\text { (n) }\end{array}$ & $\begin{array}{l}\text { Criterios } \\
\text { de inclusión }\end{array}$ & Seguimiento & $\begin{array}{c}\text { Niveles } \\
\text { de } \\
\text { vitamina D }\end{array}$ & Resultados \\
\hline $\begin{array}{l}\text { Gruson D et al. } \\
2015 \\
\text { Prospectivo }\end{array}$ & 170 & $\begin{array}{l}\text { IC NYHA II-IV } \\
\text { con FEVI } \leq 35 \%\end{array}$ & 4,1 años & $\mathrm{NE}$ & $\begin{array}{l}\text { - Correlación inversa de los niveles } \\
\text { de } 1,25(\mathrm{OH})_{2} \mathrm{D} \text { con la severidad de } \\
\text { IC (valores medios: NYHA II: } 33,3 \\
\mathrm{pg} / \mathrm{ml}, \mathrm{NYHA} \text { III: } 23,4 \mathrm{pg} / \mathrm{ml} \text {, NYHA } \\
\text { IV: } 14,0 \mathrm{pg} / \mathrm{ml} ; \mathrm{p}<0,001) \\
-1,25(\mathrm{OH})_{2} \mathrm{D} \text { y su ratio a PTH } \\
(1-84) \text { predictor independiente de } \\
\text { mortalidad cardiovascular en IC }\end{array}$ \\
\hline $\begin{array}{l}\text { Gostman let al. } \\
2012 \\
\text { Prospectivo }\end{array}$ & $\begin{array}{c}3.009 \text { con } \\
\text { IC } / 46.825 \\
\text { control }\end{array}$ & $\begin{array}{l}\text { Pacientes } 245 \text { años } \\
\text { con IC vx. } \\
\text { población control } \\
\text { con } 25 \cdot H C C \text { medida }\end{array}$ & 518 dias & $\begin{array}{l}25-\mathrm{HCC} \\
<10 \mathrm{ng} / \mathrm{ml}\end{array}$ & $\begin{array}{l}\text { - Mayor mortalidad en pacientes } \\
\text { con IC (HR=1,52; CI 95\%: } 1,21-1,92 \text {; } \\
\text { p<0,001) } \\
\text { - Reducción de mortalidad en pa- } \\
\text { cientes que recibieron suplementa- } \\
\text { ción con vitamina D (HR 0,68; } \\
\text { Cl } 95 \%: 0,54-0,85 ; \text { p } 00,0001\end{array}$ \\
\hline $\begin{array}{l}\text { Liu L et al. } \\
2011 \\
\text { Prospectivo }\end{array}$ & 548 & $\begin{array}{l}\text { IC NYHA II-IV } \\
\text { hospitalizados }\end{array}$ & 18 meses & NE & $\begin{array}{l}\text { - Por cada } 4 \mathrm{ng} / \mathrm{ml} \text { de descenso de } \\
25 \cdot \mathrm{HCC} \text {, mayor riesgo de muerte } \\
\text { por objetivo final combinado de } \\
\text { muerte por cualquier causa o insufi- } \\
\text { ciencia cardíaca y rehospitalización } \\
\text { (HR=1,09; Cl } 95 \%: 1,00-1,16 \text { ) y } \\
\text { mayor mortalidad por cualquier } \\
\text { causa (HR=1,10; Cl 95\%: 1,00-1,22) } \\
\text { - Sin efecto significativo en } \\
\text { rehospitalización por IC }\end{array}$ \\
\hline
\end{tabular}

NYHA: escala da New York Heart Association ; FEVE, fração de ejeção do ventrículo esquerdo; FC: razão de risco : IC: intervalo de confiança; NE: não especificado.

Também foi demonstrada alta prevalência de deficiência de vitamina $D$ em pacientes com IC avaliados para transplante cardíaco, bem como sua correlação inversa entre os níveis 
séricos de vitamina D e a gravidade da IC [55]. Essa relação também foi objetivada por outros grupos que usam controles sem insuficiência cardíaca [56], mesmo em pacientes mais jovens, sugerindo que existe uma associação entre IC e deficiência de vitamina $D$, independente da idade.

Portanto, embora exista uma alta prevalência de hipovitaminose D na população geral aparentemente saudável, esse déficit parece ser mais acentuado na população com IC ( Tabelas 1,2 e 3 ).

\section{RELAÇÃO ENTRE VITAMINA D E FALHA CARDÍACA}

A IC é uma doença com alta repercussão socio-sanitária, portanto, um esforço especial foi feito para prever o risco de desenvolver IC e identificar a população em risco na qual uma prevenção primária mais ativa deve ser enfatizada. Portanto, e dada a alta morbimortalidade da IC, é interessante encontrar marcadores prognósticos nessa patologia que possam prever a mortalidade. Nesse sentido, também foram feitas tentativas para confirmar a associação entre os níveis de vitamina $D$ e o risco de IC e eventos adversos por meio de estudos longitudinais.

A vitamina $D$ tem sido associada ao desenvolvimento de IC e como um fator prognóstico independente para mortalidade e morte súbita por IC em um estudo prospectivo de 3.299 pacientes caucasianos foram submetidos a cateterismo cardíaco, com seguimento médio de 7,7 anos. Quando pacientes com hipovitaminose D grave $(25 \mathrm{HCC}<10 \mathrm{ng} / \mathrm{ml})$ foram comparados com pacientes com níveis ótimos de vitamina $\mathrm{D}(25-\mathrm{HCC}>30 \mathrm{ng} / \mathrm{ml})$, foi obtida uma taxa de risco (FC) de 2,84 (IC ). 95\%: 1,20-6,74) por morte por IC e 5,05 (IC 95\%: 2,1311,97) por morte súbita. Também foi observada correlação inversa entre os níveis de propeptídeo natriurético cerebral nterminal (Nt-proBNP) e os níveis séricos de vitamina $D$ e uma associação inversa com a classe funcional da NYHA ( New York Heart Association ) [57]. Esses achados também foram posteriormente corroborados em um estudo de 2.312 indivíduos saudáveis com idade superior a 65 anos, em que foi constatado que pacientes com 25-HCC <15 ng / ml apresentavam um risco maior de 29\% (IC 95\%: 5-55\% maior) de mortalidade por qualquer causa e, para cada $10 \mathrm{ng} / \mathrm{ml}$ em que o $25-\mathrm{HCC}$ diminuiu, o risco relativo de mortalidade aumentou 9\% (IC95\%: 2-17\%) [20] .

Até agora, os dados que ligam os níveis de vitamina $D$ ao risco de desenvolver IC são conflitantes. Por um lado, não foi observada associação inequívoca dos níveis de vitamina $D$ com a incidência de IC, mas, por outro lado, sua associação com os níveis de PTH foi observada $[20,58,59]$. Assim, em um estudo com 6.469 pessoas de uma população em geral livre de 
doença cardiovascular estabelecida, com seguimento médio de 8,4 anos, após comparar pacientes com níveis de PTH $<65 \mathrm{pg} / \mathrm{ml}$ e PTH? $65 \mathrm{pg} / \mathrm{ml}$, este último apresentou $50 \%$ (IC95\%: 3-20\%) de maior risco de incidência de IC e 5,3 g (IC95\%: 2,6- 7,9 g) mais de massa ventricular esquerda determinada por RNM [58]. Da mesma forma, em uma coorte de 3.713 homens com 6079 anos com e sem doença cardiovascular, verificou-se que em pacientes com níveis de PTH>55,6 pg / $\mathrm{ml}$, havia um risco aumentado de IC de novo (HR = 1,66; IC95\%: $1.302,1)[59]$. Esses achados foram demonstrados anteriormente por Kestenbaum et al . em um estudo com 2.312 indivíduos saudáveis - 65 anos de idade, nos quais, após 14 anos de acompanhamento, descobriram que pacientes com PTH - $65 \mathrm{pg} / \mathrm{ml}$ apresentavam um risco aumentado de 30\% (IC95\%: 6- 61\%) de incidência de IC [20] .

Isso é interessante, dado que altos níveis de PTH geralmente identificam pacientes com baixos níveis de vitamina $D$, e a relação entre hipovitaminose $D$ e níveis de PTH pode ser confundida com IC. De fato, a deterioração progressiva da função renal, a inatividade física e a redução da absorção de cálcio são causas e conseqüências da hipovitaminose $D$, que por sua vez está associada ao aumento dos níveis de PTH. Portanto, à luz dos estudos apresentados anteriormente, pode-se extrapolar que foi demonstrado que existe uma associação independente de risco de insuficiência cardíaca em pacientes com baixos níveis de vitamina $D$ ou altos níveis de PTH. Isso é interessante, pois alguns autores consideram que são os níveis de PTH que predizem doenças cardiovasculares [60] .

Nos últimos anos, numerosos estudos foram publicados nos quais não apenas uma alta prevalência de deficiência de vitamina $D$ foi observada em pacientes com IC, mas também tem sido relacionada à vitamina $\mathrm{D}$ como marcador de doença mais grave e com maior taxa. de eventos adversos em pacientes com insuficiência cardíaca. Foi observada uma relação inversa entre os níveis de $25-\mathrm{HCC}$ e os níveis de peptídeo natriurético B (BNP) em pacientes com IC 20, 54 , bem como a função ventricular [61] , relatando como um marcador independente de hospitalização por IC e mortalidade [20] .

No entanto, também existe uma teoria de que a deficiência de vitamina $D$ em pacientes com IC ocorre porque esses pacientes têm uma classe funcional mais pobre, são mais fracos e, portanto, têm um estilo de vida mais sedentário; menor exposição à luz solar, o que leva a menor produção de vitamina $D$ na pele e menores níveis de vitamina $D$ [62] . Isso é questionado em diferentes estudos nos quais, após uma análise multivariada com a quantificação da atividade física, é uma associação entre os níveis de vitamina $D$ e disfunção ventricular e mortalidade por IC que permanece significativa [56] .

Por fim, também é interessante comentar a forte associação encontrada entre pacientes com fibrilação atrial e IC (uma vez que a fibrilação atrial é um importante gatilho para a exacerbação e insuficiência terapêutica da IC) em um estudo observacional que incluiu 180 pacientes. separados em dois grupos, com base no ritmo sinusal ou na fibrilação atrial permanente 56 . Os níveis plasmáticos de vitamina $D$ foram significativamente menores no 
grupo de fibrilação atrial (11,05 $\mathrm{ng} / \mathrm{ml}$ vs. $20 \mathrm{ng} / \mathrm{ml}$; $\mathrm{p}<0,001)$; os níveis de PTH foram significativamente maiores $(76,7$ vs. $55 \mathrm{pg} / \mathrm{ml}$; $\mathrm{p}<0,001)$, e o tamanho do átrio era significativamente maior $(45,03 \mathrm{~mm} / \mathrm{m} 2$ vs. $42,05 \mathrm{~mm} / \mathrm{m} \mathrm{2} ; \mathrm{p}<0,01)$ do que no grupo com ritmo sinusal. Os níveis de vitamina $D(O R=0,854 ; I C 95 \%$ : 0,805-0,907; $p<0,001)$ e tamanho atrial / superfície corporal (OR=1,077; IC95\%: 1,003-1,156; p) foram preditores independentes de fibrilação atrial.

Neste estudo, o nível de vitamina $D$ foi estabelecido como um ponto de corte preditivo para fibrilação atrial a $16,50 \mathrm{ng} / \mathrm{ml}$ (sensibilidade de 76,0\% e especificidade de $65,5 \%$, área sob a curva -AUC- = 0, 75; IC95\%: 0,67-0,82).

Em conclusão, existem evidências experimentais e clínicas que demonstram mecanismos fisiopatológicos plausíveis e uma associação direta e indireta entre a vitamina $D$ com a IC e o sistema cardiovascular. A deficiência de vitamina $D$ é muito alta em pacientes com IC e pode estar associada ao prognóstico desses pacientes $[4,6,8,10,17,19,20$, $24,30,31,35-37]$.

\section{Conflitos de interesse}

Os autores declaram não haver conflitos de interesse.

\section{REFERÊNCIAS}

1- Mosterd A, Hoes AW. Clinical epidemiology of heart failure. Heart. 2007; 93(9):1137-46.

2- Sayago-Silva I, García-López F, Segovia-Cubero J. Epidemiology of heart failure in Spain over the last 20 years. Rev Esp Cardiol (Engl Ed). 2013;66 (8):649-56.

3- Ponikowski P, Anker SD, AlHabib KF, Cowie MR, Force TL, Hu S, et al. Heart failure: preventing disease and death worldwide. ESC Hear Fail. 2014;1(1):4-25.

4- Pettifor JM. Nutritional rickets: deficiency of vitamin D, calcium, or both? Am J Clin Nutr. 2004;80:1725S-9.

5- Holick MF. Resurrection of vitamin D and rickets. J Clin Invest. 2006;116(8): 2062- 72.

6- Judd SE, Tangpricha V. Vitamin D deficiency and risk for cardiovascular disease. Am J Med Sci. 2009;338(1):40-4. 
7- Anderson JL, May HT, Horne BD, Bair TL, Hall NL, Carlquist JF, et al. Relation of vitamin D deficiency to cardiovascular risk factors, disease status, and incident events in a general healthcare population. Am J Cardiol. 2010;106(7):963-8.

8- D'Amore C, Marsico F, Parente A, Paolillo S, De Martino F, Gargiulo P, et al. Vitamin D deficiency and clinical outcome in patients with chronic heart failure: A review. Nutr Metab Cardiovasc Dis. 2017;27(10):837-49.

9- Norman AW. From vitamin D to hormone D: Fundamentals of the vitamin D endocrine system essential for good health. Am J Clin Nutr. 2008;88(2):491s-9s.

10- Camici M, Galetta F, Franzoni F, Carpi A, Zangeneh F. Vitamin D and heart. Intern Emerg Med. 2013;8(Suppl 1):5-9.

11- Simpson RU, Hershey SH, Nibbelink KA. Characterization of heart size and blood pressure in the vitamin D receptor knockout mouse. J Steroid Biochem Mol Biol. 2007;103(35):521-4.

12- Green JJ, Robinson DA, Wilson GE, Simpson RU, Westfall MV. Calcitriol modulation of cardiac contractile performance via protein kinase C. J Mol Cell Cardiol. 2006;41(2):350-9.

13- McMurray JJ, Packer M, Desay A, Gong J, Lefkowitz M. Angiotensin-neprilysin inhibition versus enalapril in heart failure. N Engl J Med. 2016;291-3.

14- Bayes-Genis A, Morant-Talamante N, Lupón J. Neprilysin and natriuretic peptide regulation in heart failure. Curr Heart Fail Rep. 2016;13(4):151-7.

15- Li YC, Kong J, Wei M, Chen Z, Liu SQ, Cao L. 1,25-Dihydroxyvitamin D3 is a negative endocrine regulator of the renin-angiotensin system. J Clin Invest.

2002;110(2):229-38.

16- Tomaschitz A, Pilz S, Ritz E, Grammer T, Drechsler C, Boehm BO, et al. Independent association between 1,25-dihydroxyvitamin D, 25-hydroxyvitamin D and the reninangiotensin system. The Ludwigshafen Risk and Cardiovascular Health (LURIC) study. Clin Chim Acta. 2010;411(17-18):1354-60.

17- Forman JP, Williams JS, Fisher NDL. Plasma 25-hydroxyvitamin D and regulation of the 
renin-angiotensin system in humans. Hypertension.

2010;55(5):1283-8.

18- Peach MJ. Renin-angiotensin system: biochemistry and mechanisms of action. Physiol Rev. 2017;57(2):313-70.

19- Li YC. Vitamin D regulation of the renin-angiotensin system. J Cell Biochem. 2003;88(2):327-31.

20- Kestenbaum B, Katz R, De Boer I, Hoofnagle A, Sarnak MJ, Shlipak MG, etal. Vitamin D, parathyroid hormone, and cardiovascular events among older adults. J Am Coll Cardiol. 2011;58(14): 1433-41.

21- Hedayat M, Mahmoudi MJ, Rose NR, Rezaei N. Proinflammatory cytokines in heart failure: Double-edged swords. Heart Fail Rev. 2010;15(6):543-62.

22- Nozaki N, Yamaguchi S, Shirakabe M, Nakamura H, Tomoike H. Soluble tumor necrosis factor receptors are elevated in relation to severity of congestive heart failure. Jpn Circ J. 1997; 61:657-64.

23- Mora JR, Iwata M, Von Andrian UH. Vitamin effects on the immunesystem: Vitamins A and D take centre stage. Nat Rev Immunol. 2008;8(9):685-98.

24- DeLuca HF. Overview of general physiologic features and functions of vitamin D. Am J Clin Nutr. 2004;80(6 Suppl):1689S-96S. [ Links ]

25- Saleh FN, Schirmer H, Sundsfjord J, Jorde R. Parathyroid hormone and left ventricular hypertrophy. Eur Heart J. 2003;2054-60.

26- Gunja-Smith Z, Morales AR, Romanelli R, Woessner JF. Remodeling of human myocardial collagen in idiopathic dilated cardiomyopathy. Role of metalloproteinases and pyridinoline cross-links. Am J Pathol. 1996;148(5):1639-48.

27- Li YY, Feng YQ, Kadokami T, McTiernan CF, Draviam R, Watkins SC, et al. Myocardial extracellular matrix remodeling in transgenic mice overexpressing tumor necrosis factor alpha can be modulated by anti-tumor necrosis factor alpha therapy. Proc Natl Acad Sci. 2002;97(23):12746-5.

28- Weber KT, Weglicki WB, Simpson RU. Macro- and micronutrient dyshomeostasis in the 
adverse structural remodelling of myocardium. Cardiovasc Res.

2009;81(3):500-8.

29- Paraguassu, Éber Coelho, et al. "Association of Bacterial Endocarditis and Buccomaxillofacial Surgery: Review of Current Medical Literature." OHDM-Oral Health and Dental Management 18 (2019).

30- Akin F, Ayça B, Köse N, Duran M, Sarı M, Uysal OK, et al. Serum vitamin D levels are independently associated with severity of coronary artery disease. J Investig Med. 2016;60(6):869-73.

31- Calistro, Lucas Cesar, et al. "Dental care in hypertense patients: systematic review." Brazilian Journal of Implantology and Health Sciences 1.6 (2019): 152-168.

32- Molinari C, Uberti F, Grossini E, Carda S, Invernizzi M, Cisari C. 1 $1 \alpha, 25-$ dihydroxycholecalciferol induces nitric oxide production in cultured endothelial cells. Cell Physiol Biochem. 2011; 27(6):661-8.

33- Grundmann M, Haidar M, Placzko S, Niendorf R, Darashchonak N, Hubel CA, et al. Vitamin $D$ improves the angiogenic properties of endothelial progenitor cells. Am J Physiol Physiol. 2012;303(9):954-62.

34- Stach K, Kälsch AI, Nguyen XD, Elmas E, Kralev S, Lang S, et al. 1 $\alpha, 25$ - dihydroxyvitamin D 3 attenuates platelet activation and the expression of VCAM-1 and MT1-MMP in human endothelial cells. Cardiology. 2011;118(2):107-15.

35- Al Mheid I, Quyyumi AA. Vitamin D and cardiovascular disease: controversy unresolved. J Am Coll Cardiol. 2017;70(1):89-100.

36- Rostand SG, Drüeke TB. Parathyroid hormone, vitamin D, and cardiovascular disease in chronic renal failure. Kidney Int. 1999;56(2):383-92.

37- Levin A, Bakris GL, Molitch M,Smulders M, Tian J, Williams LA, et al. Prevalence of abnormal serum vitamin D, PTH, calcium, and phosphorus in patients with chronic kidney disease: Results of the study to evaluate early kidney disease. Kidney Int.

2007;71(1):31-8.

38- Bischoff-Ferrari HA, Giovannucci E, Willett WC, Dietrich T, Dawson-Hughes B. Estimation of optimal serum concentrations of 25-hydroxyvitamin D for multiple health outcomes. Am J 
Clin Nutr. 2006;84(1):18-28.

39- Giustina A, Adler RA, Binkley N, Bouillon R, Ebeling PR, Lazaretti-Castro M, et al. Controversies in vitamin D: summary statement from an international conference. J Clin Endocrinol Metab. 2019;104(2):234-40.

40- Holick MF, Binkley NC, Bischoff-Ferrari HA, Gordon CM, Hanley DA, Heaney RP, et al. Evaluation, treatment, and prevention of vitamin D deficiency: An endocrine society clinical practice guideline. J Clin Endocrinol Metab. 2011;96(7):1911-30.

41- Dawson-Hughes B, Heaney RP, Holick MF, Lips P, Meunier PJ, Vieth R. Estimates of optimal vitamin D status. Osteoporos Int. 2005;16(7):713-6.

42- Holick MF. High Prevalence of vitamin D inadequacy and implications for health. Mayo Clin Proc. 2006;81(3):353-73.

43- Ross AC, Manson JAE, Abrams SA, Aloia JF, Brannon PM, Clinton SK, et al. The 2011 report on dietary reference intakes for calcium and vitamin $D$ from the Institute of Medicine: What clinicians need to know. J Clin Endocrinol Metab. 2011;96(1):53-8.

44- Glendenning P, Inderjeeth CA, Holick M. Measuring vitamin D. Clin Biochem. 2012;38(12):901-6.

45- Glendenning P, Inderjeeth CA. Vitamin D: Methods of 25 hydroxyvitamin D analysis, targeting at risk populations and selecting thresholds of treatment. Clin Biochem. 2012;45(12):901-6.

46- Kennel K, Drake MT, Hurley DL. Vitamin D deficiency in adults: when to test and how to treat. Mayo Clin Proc. 2010;85(8):752-8.

47- Holick MF. NIH public vitamin D status: measurement, interpretation and clinical application. Ann Epidemiol. 2009;19(2):73-8.

48- Palacios C, Gonzalez L. Is vitamin D deficiency a major global public health problem? J Steroid Biochem Mol Biol. 2014;144:138-45.

49- Holick MF, Chen TC. Vitamin D deficiency: a worldwide health problem. Am J Clin Nutr. 2008;87:1080-6. 
50- Groba Marco M, Mirallave Pescador A, González Rodríguez E, García Santana S, González Padilla E, Santana P S, et al. Factores relacionados con insuficiencia de vitamina D en estudiantes de Medicina de Gran Canaria. Rev Osteoporos Metab Miner. 2010;2:11-8.

51- Marwaha RK, Tandon N, Reddy DRHK, Aggarwal R, Singh R, Sawhney RC, et al. Vitamin D and bone mineral density status of healthy schoolchildren in northern India. Am J Clin Nutr. $2005 ; 82(2): 477-82$.

52- Fuleihan GE-H, Nabulsi M, Choucair M, Salamoun M, Shahine $\mathrm{CH}$, Kizirian A, et al. Hypovitaminosis D in healthy schoolchildren. Pediatrics. 2004;107 (4):e53-e53.

53- McGrath J, Kimlin M, Saha S, Eyles D, Parisi A. Vitamin D insufficiency in south- east Queensland. Med J Austr. 2001;174; 150-151.

54- Kim DH, Sabour S, Sagar UN, Adams S, Whellan DJ. Prevalence of hypovitaminosis D in cardiovascular diseases (from the National Health and Nutrition Examination Survey 2001 to 2004). Am J Cardiol. 2008;102(11):1540-4.

55- Shane E, Mancini D, Aaronson K, Silverberg SJ, Seibel MJ, Addesso V, etal. Bone mass, vitamin $D$ deficiency, and hyperparathyroidism in congestive heart failure. Am J Med. 1997;103(3):197-207.

56- Zittermann A, Schulze Schleithoff S, Tenderich G, Berthold HK, Körfer R, Stehle $P$. Low vitamin D status: A contributing factor in the pathogenesis of congestive heart failure? J Am Coll Cardiol. 2003;41(1):105-12.

57- Pilz S, März W, Wellnitz B, Seelhorst U, Fahrleitner-Pammer A, Dimai HP, etal. Association of vitamin $D$ deficiency with heart failure and sudden cardiac death in a large cross-sectional study of patients referred for coronary angiography. J Clin Endocrinol Metab. 2008;93(10):3927-35.

58- Bansal N, Zelnick L, Robinson-Cohen C, Hoofnagle AN, Ix JH, Lima JA, et al. Serum parathyroid hormone and 25hydroxyvitamin $D$ concentrations and risk of incident heart failure: The Multi-Ethnic Study of Atherosclerosis. J Am Heart Assoc. 2014;3(6):1-15.

59- Wannamethee GS, Welsh PW, Papacosta OP, Lennon L, Whincup PH, Sattar N. Elevated parathyroid hormone, but not vitamin D deficiency, is associated with increased risk of heart failure in older men with and without cardiovascular disease. Circ Hear Fail. 2014;7(5):732-9. 
60- Kamycheva E, Sundsfjord J, Jorde R. Serum parathyroid hormone levels predict coronary heart disease: The Tromso Study. Eur J Prev Cardiol. 2004;11(1):69-74.

61- Zittermann A, Ernst JB. Calciotropic and phosphaturic hormones in heartfailure. Nutr Metab Cardiovasc Dis. 2016;26(11):971-9.

62- Zittermann A, Schleithoff SS, Koerfer R. Vitamin D insufficiency in congestive heart failure: Why and what to do about it? Heart Fail Rev. 2006;11(1):25-33. 